\title{
High Energy Beam Line Based on Bending Crystal
}

\author{
V.M. Biryukov, Yu.A. Chesnokov, V.N. Greth, \\ A.A. Ivanov, V.I. Kotov, V.S. Selesnev, \\ M.V. Tarakanov, V.I. Terekhov, S.V. Tsarik \\ IHEP Protvino, 142284 Moscow Region, Russia.
}

\begin{abstract}
The possibility of abrupt bending with crystal of a beam fraction at a large angle allows to organize over a short base a nontraditional beam line for carrying out the physical experiments. At IHEP, a 150 mrad bent crystal was used this way to create a test area, to work in parallel with other set-ups consuming practically no power.
\end{abstract}

\section{I.}

Nowadays bent single crystals of silicon are applied to control high-energy particle beams. As noted in [1] the feature of the beam bending with crystals is the independence of the crystal deflector strength $\Theta=L_{D} / R_{c} \sim 0.5 \mathrm{rad}$ of the particle energy ( $L_{D}$ is dechanneling length, $R_{c}$ is the critical radius).

The possibility of abrupt bending with crystal of a beam fraction at a large angle allows to organize over a short base a nontraditional beam line for carrying out the physical experiments both in any operating accelerators and in any accelerators under construction.

At IHEP, a $150 \mathrm{mrad}$ bent crystal was used this way to create a new test area. The scheme of the crystal beam line and experimental setup is shown in Fig.1.

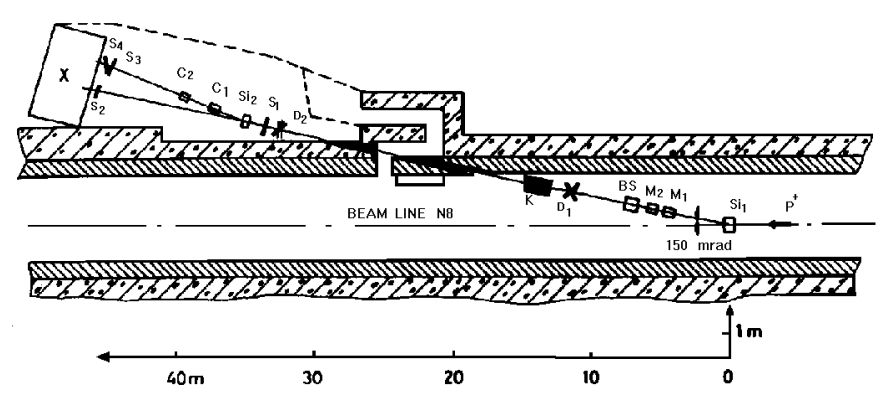

Figure. 1. The scheme of crystal beam line and experimental setup: $S i_{1}, S i_{2}$ - deflecting and testing crystals, $M_{1}, M_{2}-$ corrector magnets, $\mathrm{BS}$ - beam stopper, $D_{1}, D_{2}$ - proportional chambers, $\mathrm{K}$ - collimator, $S_{1}-S_{4}-$ scintillator countes, $\mathrm{C} 1$, $\mathrm{C} 2$ - microstrip detector stations, $\mathrm{X}$ - beam absorber.

A $100 \mathrm{~mm}$ long Si (110) crystal, placed on beam line N8 and inserted in the halo of the intense $70 \mathrm{GeV} / \mathrm{c}$ beam, has extracted $10^{6}$ protons/sec beyond the 2-meter iron-concrete shield along the $\sim 20 \mathrm{~m}$ base. Background particles emerging in the direction of a bending angle of the crystal are some tens lower on energy $\left(P_{s} \sim 3 \mathrm{Gev} / \mathrm{c}\right)$ than primary protons. It is not difficult to subtract these secondary particles and it is done with two small corrector magnets M1 and M2, collimator K and a narrow collimation hole in the iron-concrete shield of the beam line. Hereat the high quality of beam, low emittance and good stability, are achieved on the plane of the deflection ("band" beam).

In the first operating run of the new test area, the intensity of incident protons in beam line $\mathrm{N} 8$ was $2 \cdot 10^{11} \mathrm{p} / \mathrm{c}$. About $10^{10}$ particles hit the crystal. In these conditions the number of particles bent with crystal and measured by the scintillator counters was $\sim 5 \cdot 10^{5} \mathrm{p} / \mathrm{c}$. That is in accordance with a calculation. The orientation-independent component of the signal (background particles) from $10 \times 10 \mathrm{~cm}^{2}$ size counters did not exceed $3 \%$ of the channeled beam. The profiles of the deflected beam measured by chamber $D_{2}$ are shown in Fig.2. The constructed test
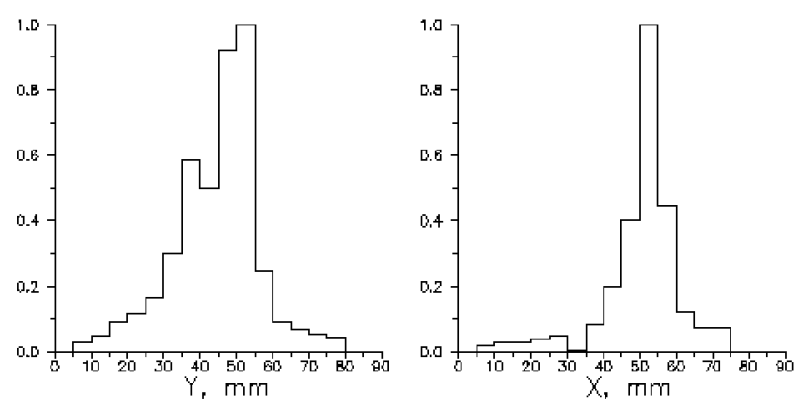

Figure. 2. Profiles of beam in horizontal (x) and vertical (y) planes.

area will allow to continue the channeling experiments. In particular, it may be used for carrying out the programs on studying the channeling properties of the crystals, for increasing the efficiency of beam deflection and focusing, and for testing the microstrip detectors. Notice that channeled beams are very suitable for a microstrip detector calibrating because they have low emittance and high stability.

The new test area consumes practically no power. It allows to work in parallel with other beam lines without any influence on the work of the other physical set-ups.

This work was supported by the Russian Fund for Basic Research.

\section{References}

[1] V.M. Biryukov, V.I. Kotov and Yu.A. Chesnokov, PhysicsUspekhi, 37937 (1994) 